\title{
Facing the Angular Stabilization of Loop-Based Artificial Magnetic Conductors Through Lumped Inductors
}

\author{
H. F. Álvarez ${ }^{1}$, M. E. de $\operatorname{Cos}^{1}$, S. García ${ }^{1}$ and F. Las-Heras ${ }^{1}$ \\ ${ }^{1}$ Area de Teoría de la Señal y Comunicaciones. Dpt. Ingeniería Eléctrica, Universidad de Oviedo. \\ Edificio Polivalente, Mod. 8. E-33203 Gijón, Asturias, Spain \\ *Humberto Fernández, E-mail: hfernandez@tsc.uniovi.es
}

\begin{abstract}
This work focuses in achieving the angular stabilization of a square loop-based AMC from a novel approach: by exploring the possibilities of introducing lumped inductors. The study is conducted considering the typical constraints an actual designer has to cope with (concerning limited size and dielectric thickness and properties). As a result, a stable $\mathrm{AMC}$ in the $5.8 \mathrm{GHz}$ band is obtained, meeting the design requirements. Due to its effectiveness, the novel developed methodology can be directly extended to enhance the angular stability of any loop-based AMC, wide spreading their applications.
\end{abstract}

\section{Introduction}

Artificial Magnetic Conductors (AMCs) are Metasurfaces (MTSs) designed to exhibit in-phase reflection (i.e to behave as a Perfect Magnetic Conductors, PMCs, which do not exist in Nature) targeting them for a wide range of marketed applications, from antenna radiation enhancement and RFID tags on metallic objects [1] to Radar Cross Section (RCS) and Specific Absorption Rate (SAR) reduction [2], to name only a few.

The AMC operation band is limited in frequency due to their inherent resonant nature. Their reflection coefficient phase continuously varies from $+180^{\circ}$ to $-180^{\circ}$, crossing $0 \circ$ at the resonance frequency. The useful bandwidth (AMC operation bandwidth) is generally defined as from +90 to $-90^{\circ}$.

Large amount of recent research effort has been devoted to cope with AMCs bandwidth limitations [3]-[4], but in comparison, few works have dealt with their angular stability enhancement [5][9], which is indeed critical for the aforementioned applications. AMCs miniaturization at low frequencies through the introduction lumped components (mainly capacitors) has been already addressed [10]. However, to the authors' best knowledge, the potential advantages of including lumped components pursuing the AMCs' angular stability enhancement has not been tackled yet.

An AMC can be considered as a metallo-dielectric Frequency Selective Surface (FSS) on a grounded dielectric substrate. Its reflectivity properties can be obtained through three-dimensional full-wave electromagnetic simulations provided a proper setup, based on a plane-wave impinging a single unit-cell with periodic boundary conditions, is defined. This kind of analysis using commercial software, such as Finite Element Method (FEM) based HFSS of Ansoft, allows analyzing the AMC reflection coefficient behavior versus frequency and so, achieving a proper design for the intended application by tailoring the periodicity, the unit-cell metallization geometry and/or the dielectric substrate. However, since the reflection coefficient can be obtained from the surface impedance, an equivalent-circuit model for the AMC surface impedance, Zs, based on lumped elements, can be used in order to get an immediate knowledge of the AMC's electromagnetic properties.

Unlike 3D full-wave simulations, the equivalent-circuit approach also provides good insight into the physical and design properties of the AMC. Such an equivalent-circuit model considers the AMC comprising a metallo-dielectric grid, with Zg impedance, on a grounded dielectric slab with Zd impedance. So that the AMC surface impedance, Zs, can be obtained as the parallel connection of $\mathrm{Zg}$ and $\mathrm{Zd}$.

Taking a glance to the literature, many works devoted to model FSS based artificial impedance surfaces can be found. However, some of them derived rather complicated formulas chasing to get a perfect fit, but losing the intuitive understanding aimed at the circuital approach and also looking scarcely attractive compared to full-wave simulations. Even taking into account that for highly reduced dielectric thickness higher order modes should be considered, as a correction factor in the equivalent-circuit model, the simplified approach used here is useful for the intended study and applications [6][7],[11].

Considering previous works [9] endorsing the advantages of loop-based AMCs over patch-based ones concerning angular stability and size reduction for a given operation frequency and dielectric substrate, this work aims at further studying the possibilities of improving the angular stability of the former ones. The new approach to achieve it relies on using lumped inductors and faces it from an actual designer point of view: with size and dielectric constraints, at a given operation frequency and for an intended application. This means that the starting point will be a nonstable loop-based AMC due to the aforementioned restraints.

The contribution is organized as follows: first the 
fundamentals of the angular stability analysis will be explained taking the reference unit-cell as basis; then the potential advantages of introducing lumped inductors concerning the angular stability enhancement will be analyzed and finally, some conclusions will be drawn.

\section{Angular stability analysis}

Once the unit-cell size (periodicity) and the dielectric substrate are fixed, the angular stability and the bandwidth of an AMC totally depend on the unit-cell metallization geometry. The larger the periodicity, the lower the resonance frequency is (which entails a reduction of the bandwidth for a fixed dielectric). The thicker the dielectric, the wider the bandwidth but the more reduced the angular stability margin. Moreover, the higher the relative dielectric permittivity is, the narrower the bandwidth, but the larger the angular stability margin.

As mentioned in the introduction, loop-based AMCs outperform patch-based ones under oblique incidence for both TE and TM polarized incident plane-waves, mainly for increasing values of the gap between cells g. Furthermore, when the analysis is constricted to loop-based unit-cells, hexagons are more stable than squares from a certain $\mathrm{g}$, due to an increase of the grid inductance in the equivalent circuit model of the AMC [9]. Taking these facts into account, a non-stable square-loop based AMC will be considered as starting point in this work, to face its angular stabilization by introducing lumped inductors.

Going a step further than a mere theoretical analysis, the study will be conducted according to the typical constraints that an actual designer has to cope with, as aforementioned in the introduction. According to it, this works aims to achieve an angularly stable loop-based AMC of $p=7.3 \mathrm{~mm}$ periodicity, on RO4003C with thickness $\mathrm{h}=1.524 \mathrm{~mm}$, with periodicity $\mathrm{p}=7.3 \mathrm{~mm}$, operating in the $5.8 \mathrm{GHz}$ frequency band, with a 7-8\% AMC operation bandwidth, and at least $3-4 \%$ stable bandwidth. The periodicity has been chosen in order to arrange 14-15 unit-cells in a $10-11 \mathrm{~cm}$ sided square (reasonable for combination with antennas and/or RCS or $\mathrm{SAR}$ reduction applications).
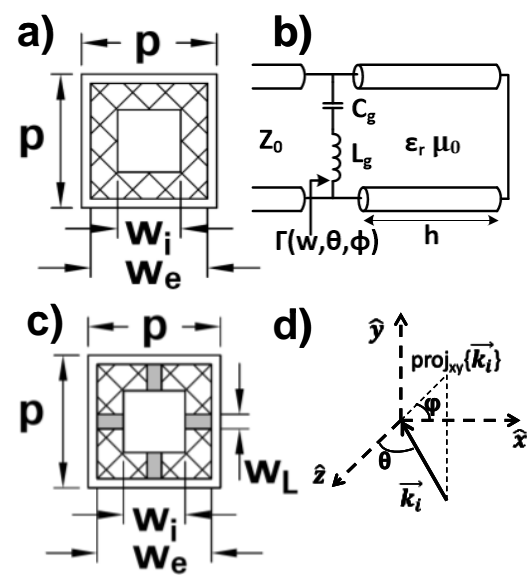

Fig. 1. (a) SLUC unit-cell geometry (top view); (b) equivalent circuit model for loop-based unit-cells; (c) SLIUC unit-cell geometry (top view); (d) coordinate system for angular stability analysis.

Fig. 1(a) shows the reference square loop-based unitcell (SLUC) geometry. Its dimensions, to fit the size and thickness imposed constraints are: $\mathrm{w}_{\mathrm{e}}=6.3 \mathrm{~mm}, \mathrm{w}_{\mathrm{i}}=3.4 \mathrm{~mm}$ and $\mathrm{g}=1 \mathrm{~mm}$.

A simple equivalent circuit to model the SLUC based AMC behavior is presented in Fig. 1(b) [6][7],[11]. $\mathrm{L}_{\mathrm{g}}$ and $\mathrm{C}_{\mathrm{g}}$ stand for the metallic strip inductance and the gap capacitance, respectively. The series connection of $\mathrm{L}_{\mathrm{g}}$ and $\mathrm{C}_{\mathrm{g}}$ accounts for the grid impedance $\left(\mathrm{Z}_{\mathrm{g}}\right)$, whereas $\mathrm{L}_{\mathrm{d}}$ represents the grounded dielectric slab inductance fully determining its impedance $\left(Z_{d}\right)$. The AMC surface impedance $Z_{s}$, comes from the parallel connection of $Z_{g}$ and $Z_{d}$, and varies with the frequency $(\omega)$, the polarization angle of the incident electric field $(\varphi)$ and the incidence angle $(\theta)$ :

$$
Z_{s}(\mathbf{w}, \boldsymbol{\theta}, \boldsymbol{\phi})=\frac{j \mathbf{w L}_{\mathbf{d}}\left(1-w^{2} L_{g} C_{g}\right)}{1-w^{2} C_{g}\left(L_{g}+L_{d}\right)}
$$

The reflection coefficient $\Gamma$ is obtained through $\mathrm{Zs}$ and $\eta_{0}$ (the free space impedance):

$$
\Gamma(\mathbf{w}, \boldsymbol{\theta}, \boldsymbol{\phi})=\frac{Z_{s}(\mathbf{w}, \boldsymbol{\theta}, \phi)-\eta_{0}}{Z_{s}(\mathbf{w}, \boldsymbol{\theta}, \boldsymbol{\phi})+\eta_{0}}
$$

The complete angular stability analysis, for both TE and TM polarized incident plane-waves, is conducted by calculating the reflection coefficient phase in simulation as follows: for each polarization angle $\varphi$, varied from $0^{\circ}$ to $90^{\circ}$ in $15^{\circ}$ steps, the incidence angle $\theta$ is varied from $0^{\circ}$ to $60^{\circ}$ also in $15^{\circ}$ steps. The phase reference plane is taken at the periodic surface and using a PEC placed in the same position as reference [4]. It is remarkable that not only the resonance frequency deviation, $\Delta \mathrm{f}$, but also the stable bandwidth $\mathrm{B}_{\mathrm{s}}$ (i. e. frequency band assuring an AMC behavior no matter the $\varphi$ and $\theta$ angles) will be considered.

The resonance frequency obtained for the SLUC AMC at normal incidence, $\mathrm{f}_{\mathrm{r}}=7.79 \mathrm{GHz}$ with a bandwidth $\mathrm{B}_{\mathrm{w}}=1508 \mathrm{MHz}(19.37 \%)$, is dramatically far from the targeted one $(5.8 \mathrm{GHz})$, due to the size and dielectric constraints. Fig. 2 shows the worst-case obtained results (narrowest $\mathrm{B}_{\mathrm{s}}$ and highest $\Delta \mathrm{f}$ ) for the SLUC based AMC: $\mathrm{B}_{\mathrm{s}}=711 \mathrm{MHz}(8.67 \%)$ for TE-polarization with $\varphi=75^{\circ}$ (Fig. 2a) and $\Delta \mathrm{f}=529 \mathrm{MHz}$ (6.57\%) for $\mathrm{TM}$ with $\varphi=0^{\circ}$ (Fig. $2 \mathrm{~b}$ ). 
In order to achieve the aimed resonance frequency while preserving the periodicity and dielectric thickness, lumped inductances, L, will be introduced, so that the total grid inductance will increase by the combination of $\mathrm{L}_{\mathrm{g}}$ and the added $\mathrm{L}$.
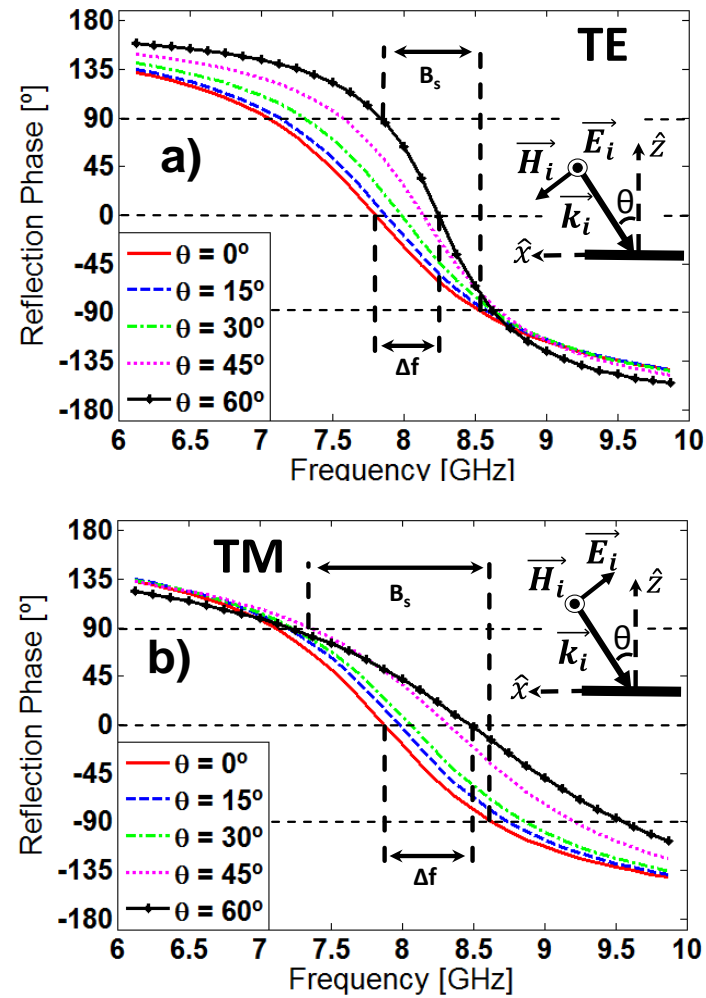

Fig. 2. Reflection coefficient phase for SLUC unit-cell. Worst cases from simulation results: (a) TE-polarization $\left(\varphi=75^{\circ}\right)$ and (b) TMpolarization $\left(\varphi=0^{\circ}\right)$.

\subsection{Comparison LUC vs LIUC based AMC concerning the angular stability}

The new unit-cell, SLIUC (Fig. 1c) is identical to the SLUC one, but for the L lumped inductances soldered one at each side of the metallization geometry. The width of the metallic strips, $1.45 \mathrm{~mm}$, had been previously chosen on SLUC so that 0805 sized lumped inductances could be soldered in SLIUC for comparison.

The formulation presented in [6]-[7],[11] is used to determine $L_{d}, L_{g}$ and $C_{g}$. Then, an estimation of the $L$ needed to achieve $\mathrm{f}_{\mathrm{r}}=5.8 \mathrm{GHz}$ can be obtained from the pole of $\mathrm{Zs}$ described in (1). $\mathrm{L}=1.8 \mathrm{nH}$ was obtained following those steps.

Fig. 3 depicts the worst-case angular stability results for SLIUC based AMC: $\mathrm{B}_{\mathrm{s}}=220 \mathrm{MHz}$ (3.82\%) for TEpolarization (Fig. 2a) and $\Delta \mathrm{f}=186 \mathrm{MHz}(3.17 \%$ ) for $\mathrm{TM}$ polarization (Fig. 2b), both with $\varphi=0^{\circ}$. First, the targeted $\mathrm{f}_{\mathrm{r}}=5.8 \mathrm{GHz}$ has been achieved in SLIUC, through a $25 \%$ decrease of the SLUC resonance frequency, fitting the size and material constraints. Furthermore, angular stability has been highly enhanced in SLIUC with a great reduction of the frequency deviation $\Delta \mathrm{f}(51.75 \%)$. The stable bandwidth, $\mathrm{B}_{\mathrm{s}}$, has decreased compared to SLUC, which was predictable since the bandwidth itself (at normal incidence) reduces as the resonance frequency decreases. Nonetheless, the $B_{s}=3.82 \%$ fits the design requirements for most of the intended applications in the $5.8 \mathrm{GHz}$ band.
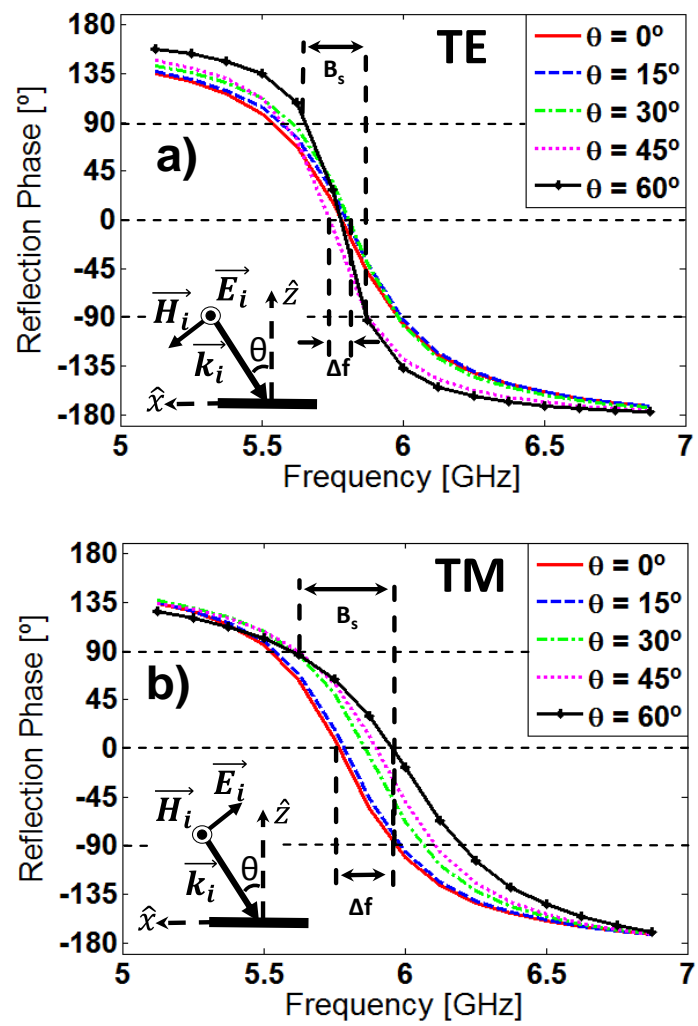

Fig. 3. Reflection coefficient phase for SLIUC unit-cell. Worst cases from simulation results: (a) TE-polarization $\left(\varphi=0^{\circ}\right)$ and (b) TM-polarization $\left(\varphi=0^{\circ}\right)$.

\section{Conclusions}

This work has faced the angular stabilization of a square loop-based AMC, SLUC, through a novel approach entailing the use of lumped inductances.

As a reward from the lumped inductances introduction, a stable SLIUC based AMC has been obtained, meeting typically imposed design constraints: operation frequency, size and dielectric substrate, bandwidth and stable bandwidth.

These results open a new approach to flexibly control the angular stability of AMC and other loop based metasurfaces, and may offer widespread applications for novel devices.

The authors continue working on studying the angular stability enhancement, further considering the dielectric thickness and properties influence, along with the application of these results for antennas performance 
improvement, due to the limitations for the proper angular stability measurement itself in anechoic chamber [12].

\section{Acknowledgements}

This work has been supported by the Gobierno del Principado de Asturias/FEDER under project GRUPIN14114 and by the Ministerio de Economía y Competitividad under project TEC2014-54005-P (MIRIIEM).

\section{References}

[1] R. C. Hadarig, M. E. de Cos and F. Las-Heras, "UHF Dipole-AMC Combination for RFID Applications," IEEE Antennas and Wireless Propagation Letters, vol. 12, no. , pp. 1041-1044, 2013.

[2] M. Mantash, A.-C. Tarot, S. Collardey, K. Mahdjoubi, "Investigation of flexible textile antennas and AMC reflectors". Int. J. Antennas Propag. 12, 1-10 (2012).

[3] L. Akhoondzadeh-Asl, J. Nourinia, C. Ghobadi, P. S. Hall, "Influence of Element Shape on the Bandwidth of Artificial Magnetic Conductors", Journal of Electromagnetic Waves and Applications Vol. 21, Iss. 7, 2007.

[4] M.E. de Cos; Y. Alvarez; F. Las-Heras, "Novel Broadband Artificial Magnetic Conductor with Hexagonal Unit Cell," IEEE Antennas and Wireless Propagation Letters, vol.10, no., pp.615-618, 2011.

[5] L. Lanuzza; A. Monorchio; D.J. Kern; D.H. Werner, "A robust GA-FSS technique for the synthesis of optimal multiband AMCs with angular stability," IEEE Antennas and Propag. Society International Symp., 2003. vol.2, no., pp. 419- 422 vol.2, 22-27 June 2003.

[6] C.R. Simovski; P.D. Maagt and I.V. Melchakova, "High-impedance surfaces having stable resonance with respect to polarization and incidence angle," IEEE Trans. on Antennas and Propagation, Vol. 53, No. 3, 908-914, March 2005

[7] O. Luukkonen; C. Simovski; G. Granet; G. Goussetis; D. Lioubtchenko; A. V. Raisanen; S. A. Tretyakov, "Simple and Accurate Analytical Model of Planar Grids and High-Impedance Surfaces Comprising Metal Strips or Patches," IEEE Transactions on Antennas and Propagation, vol.56, no.6, pp.1624,1632, June 2008

[8] Y. Ranga, L. Matekovits, K. P. Esselle and A. R. Weily, "Oblique Incidence Performance of UWB Frequency Selective Surfaces for Reflector Applications," 2011 IEEE International Symposium on Antennas and Propagation (APSURSI), pp.3170-3173, 3-8 July 2011

[9] M.E. de Cos; F. Las-Heras; "On the advantages of loopbased unit-cell's metallization regarding the angular stability of artificial magnetic conductors". Appl. Phys. A, Vol. 118, pp. 699-708. 2015.
[10]H. Liu, K. Ford, R. Langley, "Miniaturised artificial magnetic conductor design using lumped reactive components". Electron. Lett. 45(6), 294-295 (2009)

[11]I. Anderson, "On the Theory of Self-resonant Grids" Bell Syst. Techn. J., 1975, 54, pp. 1725-1731.

[12] H. Fernandez Alvarez, M. E. de Cos Gomez and F. LasHeras, "Angular Stability of Metasurfaces: Challenges Regarding Reflectivity Measurements [Measurements Corner]", IEEE Antennas and Propagation Magazine, vol. 58, no. 5, pp. 74-81, Oct. 2016. 